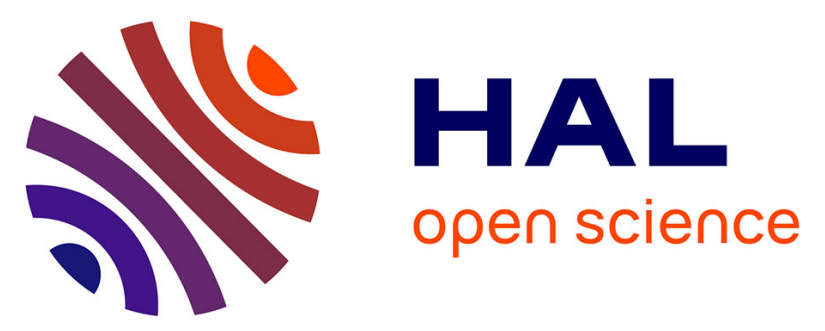

\title{
Focus sur le système endocannabinoïde et la reprotoxicité du cannabis chez la femme à l'heure du débat sur sa dépénalisation en France
}

P. Castel, P. Simon, M. M. Barbier, C. Sunyach, V. Tassistro, O. Manzoni, A.-L. Pelissier, B. Courbiere

\section{To cite this version:}

P. Castel, P. Simon, M. M. Barbier, C. Sunyach, V. Tassistro, et al.. Focus sur le système endocannabinoïde et la reprotoxicité du cannabis chez la femme à l'heure du débat sur sa dépénalisation en France. Gynécologie Obstétrique Fertilité \& Sénologie, 2020, 48 (4), pp.384-392. 10.1016/j.gofs.2020.01.024 . hal-02509031

\section{HAL Id: hal-02509031 https: / hal-amu.archives-ouvertes.fr/hal-02509031}

Submitted on 3 Apr 2020

HAL is a multi-disciplinary open access archive for the deposit and dissemination of scientific research documents, whether they are published or not. The documents may come from teaching and research institutions in France or abroad, or from public or private research centers.
L'archive ouverte pluridisciplinaire HAL, est destinée au dépôt et à la diffusion de documents scientifiques de niveau recherche, publiés ou non, émanant des établissements d'enseignement et de recherche français ou étrangers, des laboratoires publics ou privés. 


\title{
Focus sur le système endocannabinoïde et la reprotoxicité du cannabis chez la femme à l'heure du débat sur sa dépénalisation en France
}

\section{Focus on the endocannabinoid system and the reprotoxicity of marijuana in female users}

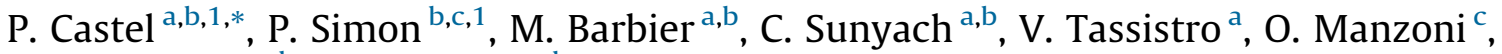 \\ A.-L. Pelissier ${ }^{\mathrm{c}, \mathrm{d}}$, B. Courbiere ${ }^{\mathrm{a}, \mathrm{b}}$ \\ a Aix-Marseille université, Avignon université, CNRS, IRD, IMBE, institut méditerranéen de biodiversité et d'écologie marine et continentale, \\ équipe biomarqueurs, environnement et santé, site Timone, 27, boulevard Jean-Moulin, 13005 Marseille, France \\ ${ }^{\mathrm{b}}$ Pôle femmes-parents-enfants, plateforme couple reproduction enfant : environnement E' risques (CREER), hôpital de La Conception, hôpital Nord, AP-HM, \\ Marseille, France \\ ${ }^{\mathrm{c}}$ Aix-Marseille université, Inserm, INMED, institut de neurobiologie de la méditerranée, 163, avenue de Luminy, 13009 Marseille, France \\ d Service de médecine légale, CHU Timone Adultes, AP-HM, Marseille, France
}

Mots clés :

Système endocannabinoïde

Grossesse

Cannabis

Reproduction féminine

Reprotoxicité

\begin{abstract}
R É S U M É
Le cannabis est le psychotrope récréatif le plus utilisé dans le monde. Les effets recherchés sont obtenus par l'action du delta9THC sur les récepteurs cérébraux du système endocannabinoïde. Le système endocannabinoïde est indispensable à l'homéostasie de l'organisme. Il est composé d'un ensemble de récepteurs, ligands endogènes et d'enzymes de son métabolisme distribués de manière ubiquitaire dans l'organisme. Chez la femme, il participe à la régulation de nombreuses étapes du processus de reproduction : régulation de l'axe hypothalamohypophysaire, ovulation, transport tubaire, implantation trophoblastique. Le delta9THC, lipophile, traverse la barrière placentaire et peut se fixer sur le système endocannabinoïde fœtal qui apparaît précocement au cours du développement. Chez l'humain, les conséquences fotales et obstétricales connues de la consommation de cannabis au cours de la grossesse sont principalement le retard de croissance et l'accouchement prématuré. Au vu des discussions actuellement menées dans plusieurs pays concernant la légalisation de la consommation de cannabis, de nouvelles recherches sont nécessaires pour en préciser les conséquences maternelles, fœtales et reprotoxiques.
\end{abstract}

\section{A B S T R A C T}

Among recreative compounds, marijuana is the most used worldwide. Delta9THC binding on brain endocannabinoid receptors drives its psychotropic effects. The endocannabinoid system (ECS) is an endogenous neurohormonal system essential for homeostasis composed of ligands, metabolic enzymes and at least 2 receptors discovered to date. In female reproduction, the ECS regulates the hypothalamicpituitary axis and many steps of the reproduction process, such as ovulation, tubal transportation and trophoblast implantation. Delta9THC can cross the placental barrier and bind to the fetal endocannabinoid system. In humans, fetal and obstetrical consequences of marijuana use during pregnancy are intrauterine growth restriction and preterm delivery. In the light of legalization projects currently reviewed in several western countries, further research should be conducted to improve knowledge on maternal, fetal and reprotoxic consequences of marijuana use during reproductive age and pregnancy.

\footnotetext{
* Auteur correspondant. Pôle femmes-parents-enfants, institut méditerranéen de biodiversité et d'écologie marine et continentale, équipe biomarqueurs, environnement et santé, site Timone, 27, boulevard Jean-Moulin, 13005 Marseille, France.

Adresse e-mail : castelpierre@orange.fr (P. Castel).

${ }^{1}$ Les deux auteurs ont équitablement contribué à l'élaboration du manuscrit.
} 


\section{Introduction}

Avec 188 millions de consommateurs réguliers estimés entre 15 et 64 ans, le cannabis est la substance psychoactive la plus consommée dans le monde. La majorité des utilisateurs sont jeunes et l'exposition se produit principalement pendant leurs années d'activité génitale [1]. Le cannabis est également le psychotrope récréatif le plus largement consommé durant la grossesse dans les pays occidentaux. En 2016, on estimait que 2,1\% des femmes enceintes en France métropolitaine avaient consommé du cannabis [2].

La découverte du principe actif du cannabis, le $\Delta 9-\mathrm{THC}$, a précédé de plusieurs décennies celle du système endocannabinoïde (SEC) par lequel ses effets sont médiés. Le système endocannabinoïde est composé de plusieurs récepteurs (les principaux étant les récepteurs $C B 1$ et $C B 2$ ), de ligands endogènes, notamment l'arachidonoyléthanolamide (AEA) et le 2-arachinonoylglycérol (2-AG) ainsi que de protéines de synthèse, de transport et de dégradation [3]. Le SEC est un système ubiquitaire majeur pour l'homéostasie de l'organisme, qui intervient dans la régulation de très nombreuses fonctions physiologiques. Chez l'animal, il a été démontré qu'il joue un rôle régulateur global sur le système reproducteur des femelles où il régule la folliculogenèse, la maturation des ovocytes et la sécrétion endocrinienne ovarienne. De plus, il joue un rôle clé au cours des étapes initiales du développement embryonnaire [4]. Chez la femme en revanche, où le rôle du SEC sur le système reproductif est difficile à confirmer expérimentalement, les études épidémiologiques concernant les effets de la consommation de cannabis sont plus discordantes. Néanmoins les preuves issues des études animales ainsi que les initiatives mondiales de légalisation ou de dépénalisation soulignent le besoin urgent de preuves scientifiques concernant les risques reprotoxiques de la consommation de cannabis. Déjà décrits sur le cerveau en développement, les effets de la consommation du cannabis sur la reproduction restent largement inexplorés [5].

L'objectif de cette revue a été d'effectuer un état des lieux des connaissances concernant les implications du SEC dans le processus de reproduction, évaluées expérimentalement chez l'animal et cliniquement chez l'humain.

\section{Méthodes}

La recherche bibliographique a été effectuée à l'aide des mots clés suivants sur Pubmed et Google Scholar pour des articles en langue anglaise entre 1964 et 2019 : " cannabinoids ", " reproduction ", " cannabis ", " marijuana ", " sexual steroids ", " lipid signalling ", "female reproduction". Nous avons examiné la pertinence des articles identifiés et sélectionné les études épidémiologiques ou expérimentales traitant des effets in vitro ou in vivo du cannabis au niveau du système reproducteur. D'autres articles jugés pertinents ont été sélectionnés à partir des bibliographies des articles recherchés.

\section{Résultats}

\subsection{Le cannabis}

Le cannabis (Cannabis sativa L.) est originaire d'Asie centrale. Il fait partie des premières plantes cultivées par l'Homme [6]. Le nom de genre du chanvre, cannabis, est employé aussi couramment pour caractériser les différentes formes sous lesquelles sont consommés la plante ou ses dérivés. Les préparations de cannabis les plus communes sont l'herbe, la résine et l'huile. L'herbe, également connue sous le nom de "marijuana ", "kif ", " weed", "beuh » etc., est un mélange de feuillets et de sommités florales des plants femelles [7]. Elle peut être consommée grâce à des pipes ou à des narguilés. La résine de cannabis, également appelée " haschich ", " hasch " ou " shit ", est une poudre brunâtre ou jaunâtre, obtenue par battage et tamisage des feuilles et des sommités florales sèches, qui est ensuite compressée pour former des barrettes ou des savonnettes [7]. La résine peut également être mélangée à du tabac.

Le cannabis contient de très nombreux composants, les cannabinoïdes représentant la principale classe (Fig. 1). Le constituant psychoactif majeur du cannabis, le $\Delta 9$-tétrahydrocannabinol (THC), a été isolé en 1964 [8]. Le cannabidiol (CBD) est un autre cannabinoïde exogène dépourvu d'activité psychodysleptique dont les effets sont anti-inflammatoire, antiépileptiques, anxiolytiques, antidépresseurs et analgésiques [9].

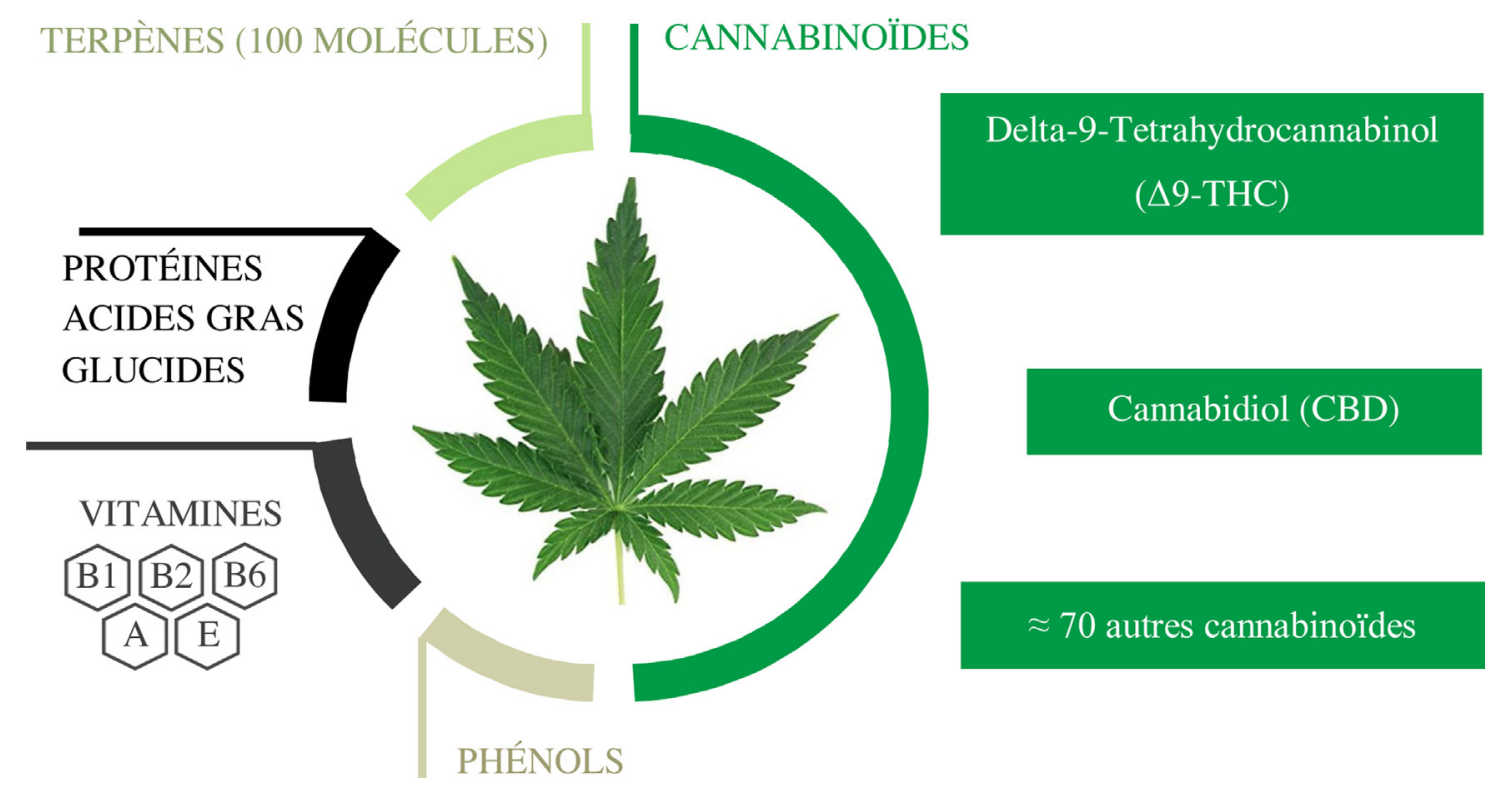

Fig. 1. Représentation graphique des composés contenus dans une feuille de $C$. sativa. 
Après une période de forte augmentation au cours des dix dernières années, la teneur en $\Delta 9$-tétrahydrocannabinol (THC) dans le cannabis sous forme d'herbe est stable autour de $10 \%$ et autour de 23-25\% pour la forme résine [10]. Les huiles et extraits, très concentrés, sont consommés par inhalation après vaporisation ou par ingestion après incorporation à une préparation alimentaire [11]. Très lipophile, le THC traverse facilement la barrière placentaire [12]. L'exposition de l'organisme fœtal est probablement plus prolongée que celle de la mère en raison d'une clairance fotale plus faible du THC. En effet, l'analyse d'échantillons de plasma fœtal et tissus fœtaux prélevés chez le primate entre 24 et 96 heures après une exposition maternelle ne retrouvait pas le principal métabolite du THC (THC-COOH). Ces données suggéraient, d'une part, que le métabolite d'origine maternelle ne traversait pas le placenta vers le fœtus et, d'autre part, que le fotus ne métabolisait pas le THC en THC-COOH [13]. Cette étude est cependant ancienne et mériterait d'être actualisée par un dosage du THC-COOH avec des techniques de dosage modernes nettement plus sensibles.

\subsection{Le système endocannabinoïde}

Le SEC est un système neuromodulateur ancestral : les récepteurs cannabinoïdes semblent communs aux vertébrés [14]. Il joue un rôle important dans le développement du système nerveux central (SNC), la plasticité synaptique et la réponse aux agressions environnementales (ex. : stress) et endogènes avec son implication dans la modulation de la peur et de l'anxiété [15]. Les recherches sur le mécanisme d'action du THC ont mené à la découverte de ses principales cibles moléculaires physiologiquement stimulées par des ligands lipidiques endogènes, les endocannabinoïdes (eCB) [16].

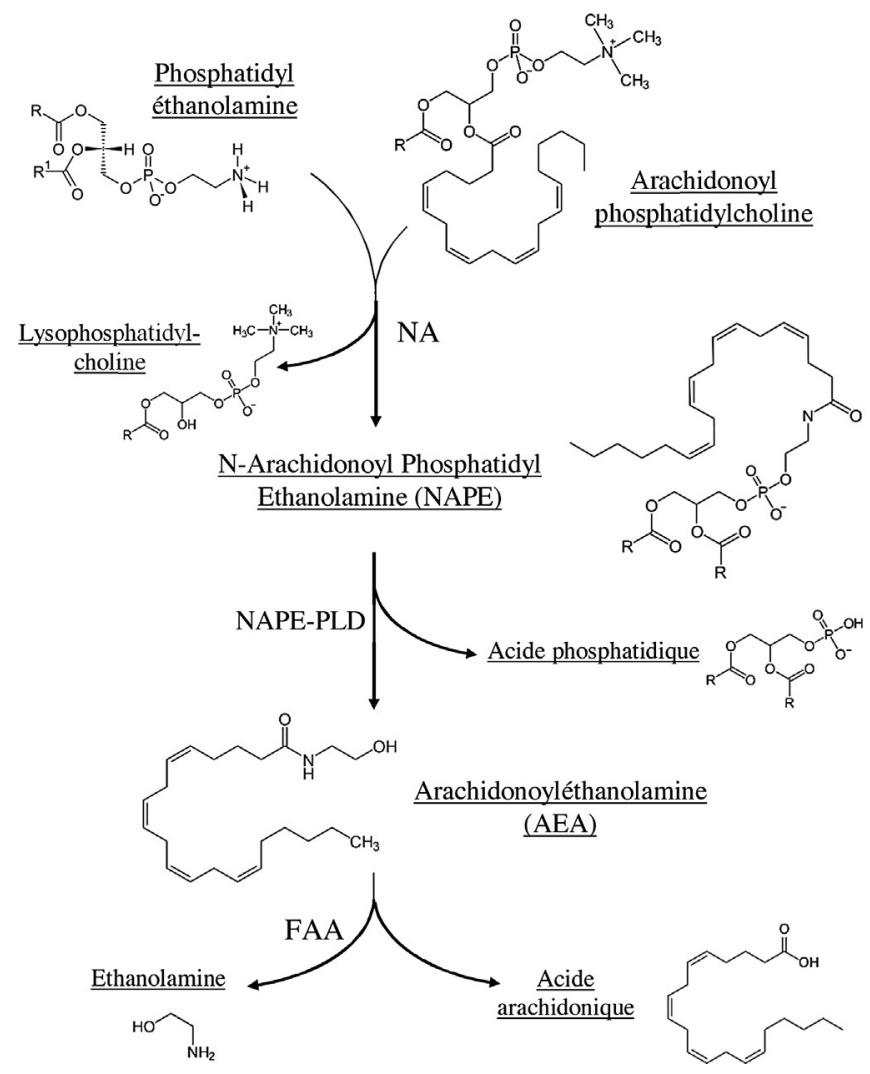

\subsection{Synthèse des endocannabinoïdes}

Les premiers endocannabinoïdes découverts et caractérisés ont été l'anandamide (arachidonoyl-éthanolamine ou AEA) et le 2-arachidonoyl-glycérol (2-AG) [17]. Les eCBs sont synthétisés à la demande à partir de précurseurs lipidiques présents dans la membrane cellulaire après stimulation par dépolarisation. Quatre voies de biosynthèse de l'anandamide ont été décrites [18]. La voie principale synthétise l'anandamide à partir de son précurseur membranaire, la N-arachidonoyl phosphatidylethanolamine (NAPE) par clivage par une phospholipase D (NAPE-PLD) (Fig. 2). La diffusion de l'anandamide à travers la bicouche lipidique se fait de manière passive ou active au travers du transporteur EMT (Endocannabinoid Membranal Transporter) [19]. Pour le 2-AG, la principale voie de biosynthèse implique l'hydrolyse du phosphatidylinositol par la phospholipase C (PLC), produisant du 1,2-diacylglycérol (DAG), qui est ensuite transformé en 2-AG (Fig. 2) [20].

\subsection{Les récepteurs endocannabinoïdes types 1 et 2 (CB1 et CB2)}

Ces récepteurs appartiennent à la superfamille des récepteurs à 7 domaines transmembranaires couplés aux protéines $G$ [21]. L'activation des récepteurs CB1 et CB2 entraîne l'inhibition de l'AMP cyclique, l'activation de la voie des MAP kinases, et le contrôle de la perméabilité de canaux ioniques calciques et potassiques [21] (Fig. 3). Ces voies de transduction aboutissent à la régulation de grandes voies de signalisation intracellulaires comme (PTEN)PI3K-Akt-mTOR impliquée dans la croissance et la prolifération cellulaire, les MAP kinases MEK-ERK dans la division cellulaire, JNK, Bax-Bcl dans le processus d'apoptose [22,23].

La signalisation cannabinoïde est complexifiée par des différences pharmacodynamiques entre les ligands. Par exemple,
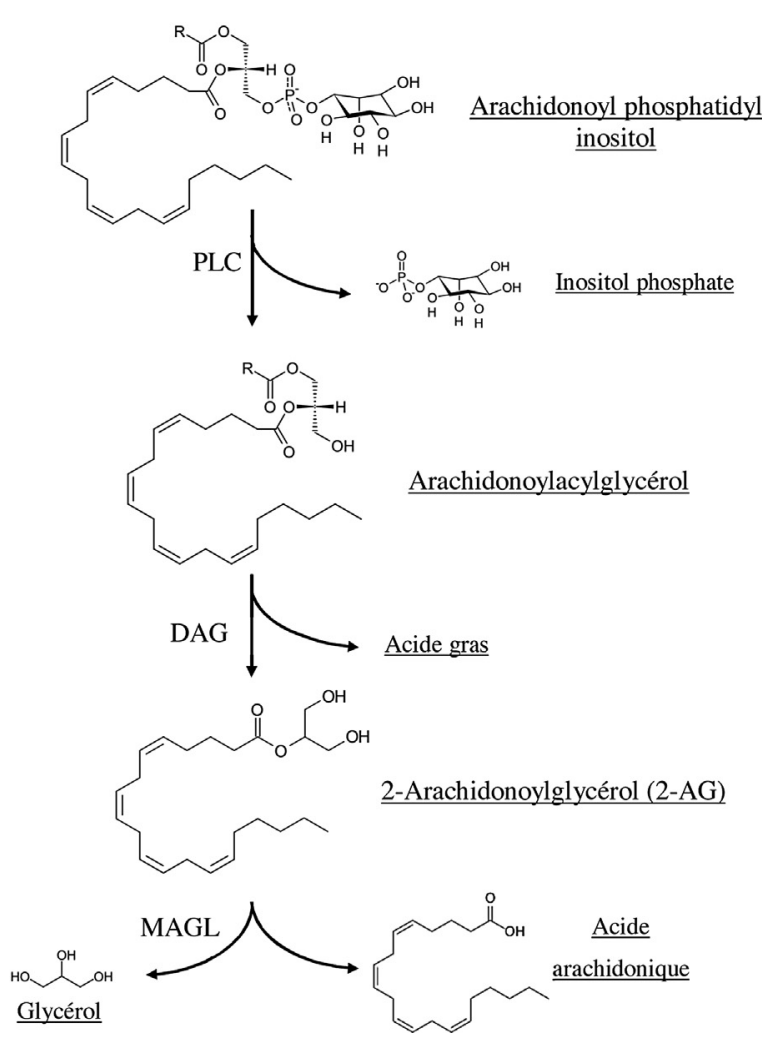

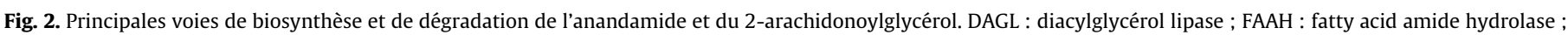
MAGL : monoacylglycérol lipase ; NAT : N-acyl transférase ; PLC : phospholipase C ; PLD : phospholipase D. 


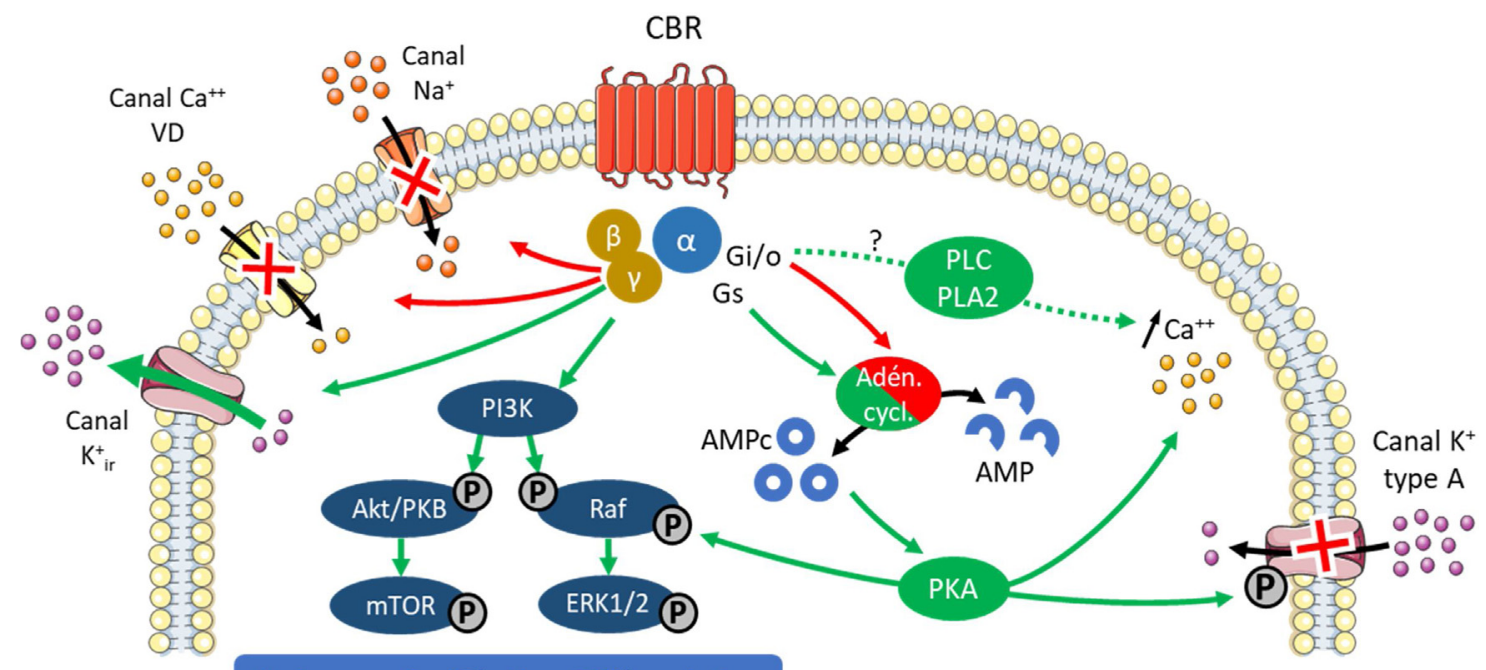

Croissance Prolifération Différenciation

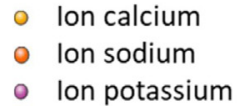

- Ion calcium

- Ion potassium
Sous unités de protéine $\mathrm{G}$ hétérotrimérique
(P) Groupe phosphoryl

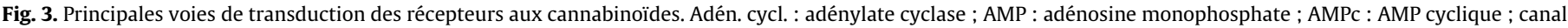

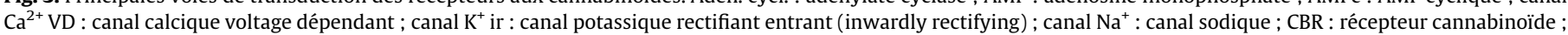
Gi : protéine G inhibitrice ; Gs : protéine G stimulatrice ; PKA : protéine kinase A ; PLA2 : phospholipase A2 ; PLC : phospholipase C.

l'anandamide et le $\Delta 9$-THC agissent comme des agonistes partiels de CB1 et CB2 alors que le 2-AG est un agoniste complet des CB1 et CB2 [24]. Des particularités pharmacodynamiques encore plus complexes comme le phénomène de sélectivité fonctionnelle ont été démontrées sur les récepteurs $C B$. Deux ligands avec des constantes d'affinité proches peuvent activer préférentiellement une voie de transduction plutôt qu'une autre et entraîner des effets spécifiques selon le type cellulaire [25].

Les études pharmacodynamiques ont été menées in vitro sur des cellules en culture ou au sein du système nerveux central. À notre connaissance, il n'existe pas d'étude évaluant la pharmacodynamie des cannabinoïdes dans l'appareil reproducteur humain.

\subsection{Dégradation des endocannabinoides}

L'anandamide est rapidement hydrolysée par une aminohydrolase (FAAH) en éthanolamine et en acide arachidonique [26]. L'hydrolyse du 2-AG en acide gras et glycérol est principalement due à trois enzymes : la monoacylglycérol lipase (MAGL) et les lipases ABDH6 et 12 (Fig. 2) [20].

\section{Consommation de cannabis - Données épidémiologiques}

Le cannabis est le psychotrope illicite le plus largement consommé durant la grossesse dans les pays occidentaux. En 2016, il a été estimé que 2,1 \% des femmes enceintes en France métropolitaine avaient consommé du cannabis [2]. Parmi elles, environ la moitié ont déclaré une consommation régulière ( 3 fois par mois et plus). Aux États-Unis, la prévalence de la consommation de cannabis auto-déclarée est autour de $4 \%$ dans la plupart des études et augmente entre 7 et $10 \%$ chez les jeunes femmes célibataires de 18 à 25 ans [27,28]. Ces chiffres augmentent entre 15 et $28 \%$ parmi les jeunes femmes sans emploi, ou appartenant à un groupe ethnique minoritaire [29,30].

Pendant la grossesse, la plupart des études existantes sont rétrospectives et basées sur l'auto-déclaration pour déterminer l'exposition et le niveau de consommation. Dans la revue de la littérature de Chiandetti et al., 3 études visaient à comparer les questionnaires par rapport aux biomarqueurs d'exposition au THC dans le liquide méconial et les cheveux maternels. La prévalence de consommation autodéclarée était de $2,9 \%$ et augmentait entre 4 à 12,4\% lorsqu'elle était évaluée par des biomarqueurs [31]. De même, dans une population de femmes enceintes d'un centre californien, la prévalence de consommation évaluée par les tests toxicologiques était deux fois plus élevée que lorsqu'elle était auto-rapportée [32]. Ces données illustrent le biais déclaratif entraînant une sousestimation de la prévalence de consommation dans les études épidémiologiques n'utilisant pas de biomarqueurs d'exposition.

Il a aussi été rapporté que 34 à $60 \%$ des consommatrices de cannabis poursuivaient leur consommation pendant la grossesse et la présence d'un partenaire consommateur est l'un des facteurs les plus fortement associés à la poursuite de la consommation de cannabis pendant la grossesse ( $O R=38,56[6,14 ; 58,88]$ ) $[29,33]$. De nombreuses femmes estiment que la consommation de cannabis est relativement sûre pendant la grossesse et moins coûteuse que le tabac $[30,34]$. La proportion de femmes enceintes ayant récemment consommé du cannabis et évaluant cette consommation comme "sans aucun risque " est ainsi passée de 25,8 à 65,4 \% entre 2005 et 2015 [35].

\section{Système endocannabinoïde et appareil reproducteur féminin : impact du cannabis}

\subsection{Localisation et rôle du système endocannabinoïde dans l'appareil reproducteur}

L'ensemble du SEC est présent dans l'ovaire humain et les récepteurs $\mathrm{CB} 1$ et $\mathrm{CB} 2$ ont été détectés dans les ovocytes à tous les stades de maturation, mais également au niveau des cellules de la thèque et de la granulosa [36]. Pendant la vie génitale, le système endocannabinoïde était exprimé de manière différentielle en fonction des stades de maturation folliculaire. Le récepteur CB1 a été identifié dans l'ovocyte jusqu'au stade secondaire avec antrum tandis que le récepteur CB2 l'a été à tous les stades, ainsi que dans 
le corps jaune. Dans la granulosa, les deux récepteurs étaient exprimés à tous les stades et la FAAH apparaît à partir du stade secondaire. Il en est de même pour la NAPE-PLD, dont l'expression était également retrouvée au stade primordial. Les niveaux d'AEA présentaient une corrélation positive faible avec l'avancement de la maturation $\left(R^{2}=0,13, p=0,03\right)$ [36]. Ces profils d'expression variables en fonction des stades de maturation font suspecter une participation du SEC dans la régulation de la maturation folliculaire. Le SEC est également présent dans les trompes et dans l'utérus, où les niveaux mesurés y sont plus élevés que dans le cerveau [37].

\subsection{Impact du cannabis sur l'axe hypothalamohypophysaire}

La sécrétion de Gonadotrophin Releasing Hormone (GnRH) est régulée par l'activation des récepteurs $\mathrm{CB} 1$ situés dans la zone préoptique de l'hypothalamus [38]. Au niveau hypophysaire, la présence de récepteurs $C B 1$ suggère un effet sur la sécrétion de l'hormone lutéinisante (LH) et l'hormone folliculo-stimulante (FSH) [39]. Il est intéressant de noter que l'expression et la distribution de FAAH, NAPE-PLD, CB1 et CB2 dans les tissus reproducteurs féminins varient également avec le cycle menstruel, ce qui suggère que l'expression du SEC est corrélée aux fluctuations hormonales [40].

Les résultats d'études chez les rongeurs et les primates suggèrent que l'administration aiguë de THC inhibe la libération par l'hypothalamus de la Gonadotrophin Releasing Hormone (GnRH), bloquant ainsi la libération de FSH et de LH $[41,42]$. Chez cinq femelles singe rhésus, l'administration quotidienne de THC par voie sous-cutanée $(2,5 \mathrm{mg} / \mathrm{kg}$ de $\Delta 9 \mathrm{THC}$, équivalent à 5-6 joints) pendant la phase folliculaire inhibait l'ovulation chez 4 d'entre elles. Au cours du cycle suivant et en l'absence de nouvelle exposition, 2 femelles sur 5 présentaient à nouveau un cycle anovulatoire et la durée du cycle était modifiée chez tous les animaux [43].

Chez l'humain, les études sont plus contradictoires mais suggèrent aussi qu'il existe un lien entre la consommation de cannabis et les perturbations du cycle menstruel. Pour Mueller et al., les consommatrices avaient un risque légèrement plus élevé de cycles anovulatoires (RR $1,7[1,0 ; 3,0]$ ) [44]. Au cours de procédures de fécondation in vitro, la consommation de cannabis dans l'année précédente était associée à une réduction de $25 \%$ du nombre d'ovocytes récupérés au cours de la ponction [45].

Enfin, l'exposition aiguë au cannabis peut également entraîner une perturbation des niveaux de LH. L'inhalation en une seule séance d'une cigarette de $1 \mathrm{~g}$ dosée à 1,8\% de THC était suffisante pour diminuer de $30 \%$ la LH plasmatique lorsque celle-ci était consommée durant la phase lutéale du cycle menstruel [46]. Or, la suppression de la LH pendant la phase lutéale précoce peut avoir des effets délétères sur les grossesses précoces en réduisant la production ovarienne de progestérone.

L'ensemble de ces données montre que le THC exerce des effets négatifs sur la reproduction en réduisant la sécrétion de GnRH. Ceci aboutit à des altérations du contrôle de la sécrétion de LH et FSH et à un risque d'anovulation. Ces effets semblent toutefois atténués chez les utilisatrices chroniques où apparaît un phénomène de tolérance $[47,48]$.

\subsection{Implication du système endocannabinoide dans le transport tubaire de l'embryon}

Après la fécondation, le transport embryonnaire vers l'utérus est activement dirigé par les contractions de l'oviducte via un processus régulé par le récepteur $\mathrm{CB} 1$. En effet, chez des souris dont le récepteur $\mathrm{CB} 1$ était génétiquement inactivé ou en cas de double inactivation CB1-CB2, mais pas en cas d'inactivation seule de CB2, $35 \%$ des embryons étaient retenus dans l'oviducte. Le maximum d'effet était observé par l'antagonisation pharmacologique du récepteur CB1 chez des souris de phénotype sauvage (63\% de rétention). Le mécanisme proposé par les auteurs était l'existence d'un tonus adrénergique CB1-dépendant régularisant les contractions de l'oviducte et la synchronisation de l'ouverture de l'ostium tubaire [49]. C'est la perte de l'activité endocannabinoïde basale qui entraînerait ces effets délétères, puisque de faibles taux de FAAH et des taux élevés d'AEA dans l'oviducte ont aussi été associés à un mauvais développement embryonnaire et à leur arrêt dans l'oviducte [50].

Chez la femme, Gebeh et al., ont prospectivement étudié l'expression du SEC au niveau des trompes des femmes ayant une grossesse ectopique comparativement à des femmes non enceintes en phase folliculaire et en phase lutéale [51]. Les résultats montraient une diminution de l'activité de la FAAH, de l'expression des récepteurs $C B 1$ et une augmentation du taux d'AEA dans le groupe de grossesse ectopique comparé au groupe contrôle en phase lutéale. Une importante mais inévitable limite résidait dans l'évaluation du système endocannabinoïde du groupe témoin dans lequel les femmes n'étaient pas enceintes. Cette étude semble néanmoins mettre en évidence que chez la femme également, une dysrégulation du SEC tubaire affecterait le transport embryonnaire et entraînerait un ralentissement de la progression de l'embryon qui se trouve toujours dans la trompe au moment de l'implantation. À ce jour toutefois, aucune étude clinique n'a étudié l'association entre la consommation de cannabis et la survenue d'une grossesse extra-utérine.

\subsection{Implication du système endocannabinoïde dans les premières divisions embryonnaires}

Au cours du transport tubaire, l'embryon est le siège des premières divisions cellulaires. Chez des embryons de souris déficients en récepteurs CB1, Wang et al. ont observé un retard de développement embryonnaire : au $4{ }^{\mathrm{e}}$ jour, $64 \%$ d'entre eux avaient atteint le stade blastocyste contre $87 \%$ dans le groupe témoin phénotype sauvage. Des résultats similaires ont été obtenus chez des souris de phénotype sauvage exposées à un antagoniste sélectif du récepteur CB1 [49]. À l'inverse, l'hyperactivation du système endocannabinoïde est aussi délétère pour le développement embryonnaire in vitro. Pour Paria et al., l'exposition d'embryons de souris, au stade 2 cellules, avec un agoniste du SEC (AEA, THC, CBD, CP55,940, WIN55,212) entraînait de manière dose-dépendante un défaut de progression vers le stade blastocyste sauf chez les souris exposées au CBD [4].

\subsection{Implication du système endocannabinoide dans l'implantation}

Après l'ovulation, pendant la fenêtre implantatoire, l'implantation d'un blastocyste nécessite d'avoir un endomètre dont les cellules stromales ont subi une série de changements morphologiques appelée décidualisation [52]. Des niveaux accrus d'AEA exercent des effets toxiques sur les cellules déciduales par activation du récepteur CB1. De fait, l'implantation ne se produit physiologiquement que dans les régions où les niveaux de $\mathrm{CB} 1$ et d'AEA sont faibles [53]. Maccarrone et al. ont montré qu'un faible niveau d'activité de la FAAH plasmatique (potentiellement corrélé à des niveaux élevés d'AEA) était associé à la survenue de fausses couches dans les 10 jours suivants [54]. Parallèlement, pour ElTalatini et al., les concentrations d'AEA plasmatique les plus faibles coïncidaient avec la fenêtre d'implantation présumée [55].

\subsection{Implication du système endocannabinoïde sur la placentation}

Le développement du placenta humain est un événement hautement coordonné. Le placenta est essentiellement constitué de quatre populations cellulaires : cytotrophoblastes, syncytiotrophoblastes, trophoblastes extra villeux et cellules trophoblasti- 
ques géantes qui expriment l'ensemble du SEC. Une signalisation endocannabinoïde appropriée est nécessaire pour un développement placentaire correct. Les eCBs induisent l'apoptose des cellules cytotrophoblastiques humaines et altèrent leur syncytialisation par l'activation des récepteurs cannabinoïdes [56,57]. In vitro, le THC a un double effet dans les cultures primaires de lignées cytotrophoblastiques et syncytiotrophoblastiques. Les faibles doses de THC favorisaient une augmentation de l'activité métabolique cellulaire par une action antioxydante, alors que des concentrations plus élevées diminuaient la viabilité cellulaire [58]. Ces résultats étaient également retrouvés par Almada et al. : l'exposition à un agoniste synthétique du THC de cellules cytotrophoblastiques issues de placenta humain entraînait leur apoptose selon une relation dose-dépendante [59].

\subsection{Implication du système endocannabinoïde dans les fausses couches spontanées}

Dans les méta-analyse de Gunn et Conner, il n'était pas retrouvé d'association entre une exposition au cannabis in utero et un risque de fausses couches spontanées $[60,61]$. L'étude de Coleman-Cowger et al. s'est intéressée aux conséquences de l'usage conjoint de cannabis et de tabac sur le développement fotal, comparativement aux effets liés à l'usage unique de l'un ou l'autre [62]. Les résultats montraient un risque de fausse couche spontanée 12 fois supérieur à celui du groupe non consommateur (OR 12,1 [1,03;141,8]). Ces résultats contradictoires peuvent s'expliquer par la difficulté à comparer les niveaux de consommation. En effet, la prévalence de la consommation en cannabis a triplé depuis 1992 et la teneur en THC dans les produits de consommation ont quasiment triplé pour atteindre $23 \%$ dans la résine et $11 \%$ dans l'herbe [10].

Chez des femmes présentant des fausses couches à répétition, des niveaux de FAAH ont été retrouvés à des taux plus élevés dans le trophoblaste extra villeux que dans le trophoblaste de grossesses normales, ce qui laisse supposer que le niveau d'anandamide y est plus faible [63]. Bien que ces résultats semblent contradictoires avec ceux de Maccarrone et al. [54], ils semblent souligner l'importance d'un tonus endocannabinoïde et de la finesse de régulation de ces étapes de la reproduction par le SEC, où l'hypercomme l'hypo-activation du SEC seraient délétères.

\subsection{Implication du système endocannabinoïde sur la croissance fœtale}

Seule l'étude de la génération $\mathrm{R}$ a évalué de façon prospective la croissance fotale dans le cas d'une exposition au cannabis [64]. Une restriction dose dépendante de la croissance fœtale a été observée chez 9778 femmes exposées : chez les fotus exposés dès le début de la grossesse de façon discontinue, la croissance était réduite de 11,2 grammes par semaine, tandis que chez les fœtus exposés de façon continue au cannabis, elle l'était de 14,4 grammes par semaine [64]. La méta-analyse de Gunn et al. retrouvait une réduction de 109,4 $\pm 70,7 \mathrm{~g}$ à la naissance [60]. Les conséquences cliniques de ces restrictions de croissance assez minimes sont toutefois difficiles à apprécier. Pour Gunn et al., il existait cependant une association positive entre la consommation de cannabis au cours de la grossesse et la fréquence de poids de naissance inférieurs à 2500 grammes (OR 1,77 [1,04;3,01]) [60]. Cette association n'était retrouvée par Conner et al. que dans la sous-population des femmes ayant déclaré une consommation modérée à forte de marijuana au moins une fois par semaine (OR 1,90 [1,44;2,45]) [61].

\subsection{Impact du cannabis sur le risque d'accouchement prématuré}

Trois études prospectives ont démontré un risque accru de naissances prématurées chez les femmes consommant du cannabis pendant la grossesse (Tableau 1). La survenue d'un

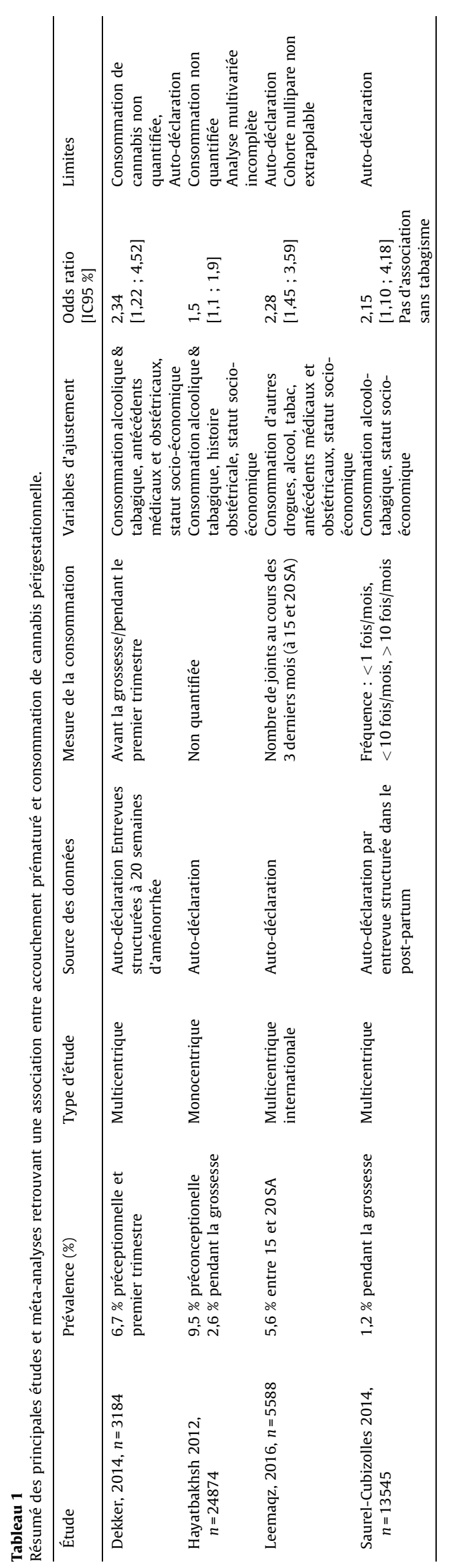


accouchement prématuré était associée à la consommation de cannabis pendant les deux premiers trimestres pour Leemaqz et al. (OR $2,28[1,45 ; 3,59]$ ) et Hayatbakhsh et al. (OR $1,5[1,1 ; 1,9]$ ) ou en cas de consommation après 20 semaines d'aménorrhée (OR 5,44 $[2,44 ; 12,11])[65,66]$. En France, Saurel-Cubizolles et al. ont constaté un risque accru de naissances prématurées spontanées chez les femmes qui consommaient du cannabis (OR 2,15 [1,10 ; 4,18]) [67]. Toutefois, cette association n'était plus significative lorsque la consommation de tabac était prise en compte dans le modèle.

La consommation de cannabis avant la grossesse était également associée à un surrisque d'accouchement prématuré pour Dekker et al. (OR 2,34 [1,22; 4,52]) [68]. Plusieurs autres études de cohortes prospectives et analyses secondaires ne fournissent pas de réponse définitive concernant le risque de naissance prématurée et la consommation de marijuana [60,6972]. Dans l'ensemble de ces études cliniques, le tabac est probablement un facteur confondant majeur responsable des effets observés en analyse univariée. Comme pour la croissance fœtale et le poids de naissance, Conner et al. ne retrouvaient pas d'association dans la population globale entre la prématurité et la consommation de cannabis après ajustement (OR 1,08 [0,82 ; $1,43])[61]$.

Les données concernant la consommation de cannabis pendant la grossesse et la survenue d'une naissance prématurée semblent discordantes, fortement affectées par la présence de facteurs de confusion (tabac, précarité) et la difficulté à quantifier la consommation. La possibilité d'un effet dose-dépendant semble toutefois possible et semble retrouvé pour deux évènements obstétricaux ayant un impact sur la morbidité néonatale l'accouchement prématuré ainsi que le petit poids de naissance. Concernant la morbidité néonatale. Gunn et al. [60] ont mis en évidence une augmentation du taux d'admission en unités de soins continus des nouveau-nés après exposition prénatale au cannabis (OR 2,02 [1,04; 3,01]). Ces résultats sont concordants avec ceux de l'étude rétrospective unicentrique de Warshak et al. (OR 1,54 $[1,14 ; 2,07])[73]$. En revanche, ce résultat n'a pas été retrouvé dans les études de Conner et al. (pooled OR $1,41[0,99 ; 2,00]$ ) ni de Metz et al. $(1,91[0,88 ; 4,16])[61,74]$.

\section{Conclusion}

Les 20 dernières années ont été marquées par des découvertes concernant l'implication du système endocannabinoïde dans la régulation des processus de reproduction. Chez l'animal, le SEC semble impliqué dans la régulation de toutes les étapes du processus de reproduction, de la folliculogenèse jusqu'à l'implantation embryonnaire et même la durée de gestation (Fig. 4). Il apparaît que le concept de tonus endocannabinoïde décrit par Sun et Dey est nécessaire à un processus de reproduction physiologique et que les déséquilibres en faveur d'une hypo- ou d'une hyperactivation du SEC sont délétères [75]. Toutefois, la plupart des études expérimentales étant menées chez le rongeur, l'extrapolation des résultats chez l'humain est difficile. Par ailleurs, les conséquences cliniques de la consommation de cannabis chez la femme sont difficiles à évaluer en raison de l'illégalité de la substance dans de nombreux pays, de l'inégalité de teneur en delta9THC, de l'existence de facteurs de confusion et des problématiques de sous-déclaration de la consommation. Au vu de l'importance du SEC dans la régulation de nombreux processus physiologiques de la reproduction mais aussi extra-reproducteurs, il est également possible que l'exposition in utero ait des conséquences à long terme sur la descendance. Cet aspect, maintenant bien connu dans les neurosciences, demeure pourtant peu étudié dans le domaine de la fertilité [76].

Ainsi, le débat actuel sur la légalisation de la consommation du cannabis semble s'accompagner d'une banalisation de sa consommation, en particulier chez les femmes en âge de procréer et chez les femmes enceintes. Il nous apparaît ainsi indispensable de mieux caractériser les effets d'une consommation de cannabis sur la fertilité et pendant les 1000 premiers jours de développement afin de mieux pouvoir délivrer des messages de prévention ciblés de santé publique [77].

\section{Financements}

Ce travail a bénéficié d'une aide du gouvernement français au titre du Programme investissements d'avenir, initiative d'excellence d'Aix-Marseille Université - A*MIDEX.

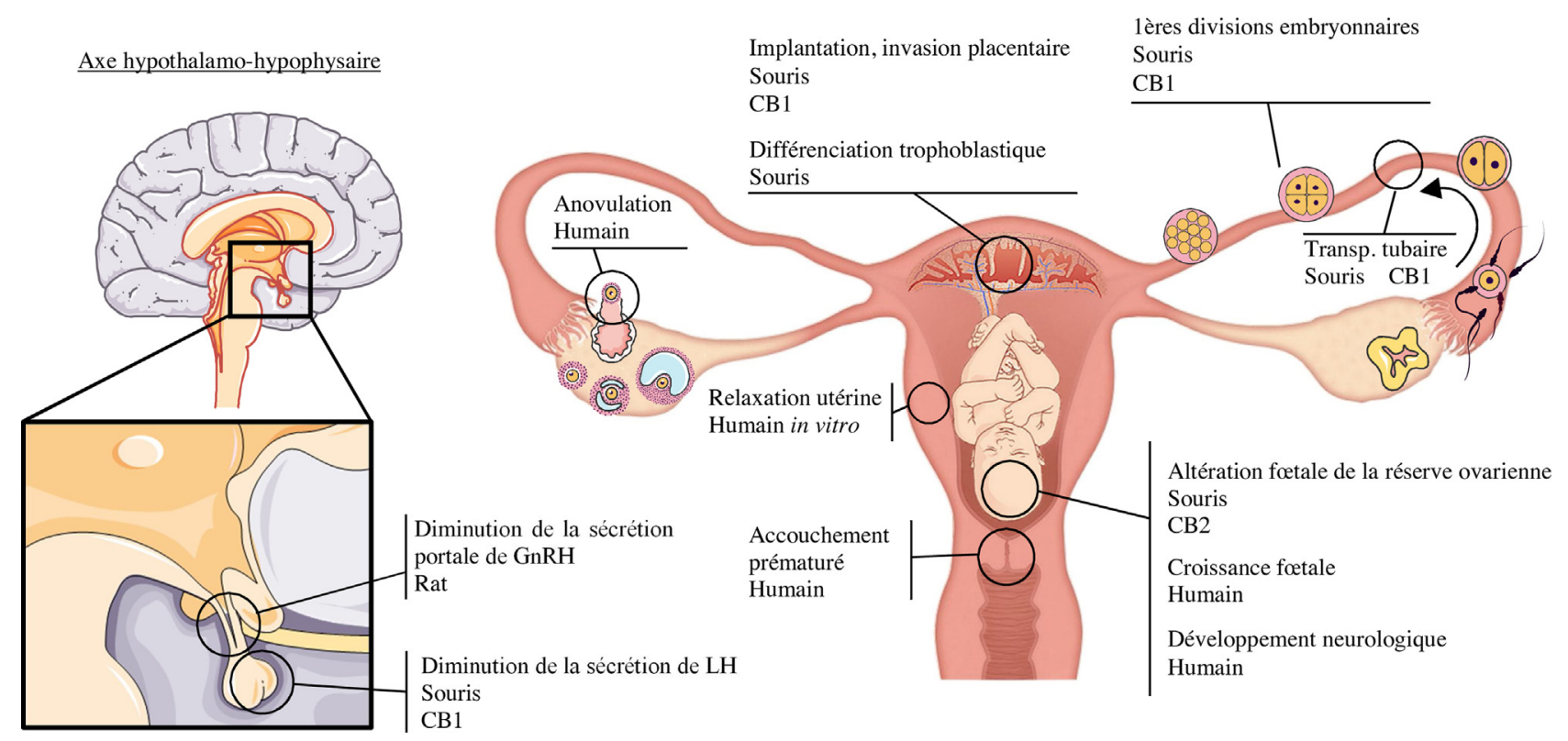

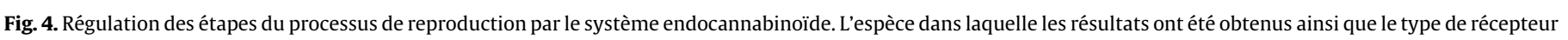
endocannabinoïde impliqué, s'il était connu, ont été précisés. 


\section{Déclaration de liens d'intérêts}

Les auteurs déclarent ne pas avoir de liens d'intérêts.

\section{Références}

[1] United Nations Office on Drugs and Crime. World Drug Report 2019; 2019.

[2] DRESS I. Enquête nationale périnatale 2016. Les naissances et les établissements, situation et évolution depuis 2010 - Ministère des Solidarités et de la Santé; 2016.

[3] Maccarrone M, Dainese E, Oddi S. Intracellular trafficking of anandamide: new concepts for signaling. Trends Biochem Sci 2010;35:601-8. http://dx.doi.org/ 10.1016/j.tibs.2010.05.008.

[4] Paria BC, Ma W, Andrenyak DM, Schmid PC, Schmid HH, Moody DE, et al Effects of cannabinoids on preimplantation mouse embryo development and implantation are mediated by brain-type cannabinoid receptors. Biol Reprod 1998:58:1490-5.

[5] Tortoriello G, Morris CV, Alpar A, Fuzik J, Shirran SL, Calvigioni D, et al. Miswiring the brain: $\Delta 9$-tetrahydrocannabinol disrupts cortical development by inducing an SCG10/stathmin-2 degradation pathway. EMBO J 2014;33: 668-85. http://dx.doi.org/10.1002/embj.201386035.

[6] Newton DE. Marijuana: a reference handbook. ABC-CLIO; 2013

[7] Paczesny M. Cannabis sativa L. : étude botanique et chimique : propriétés médicales et état des lieux sur la réglementation; 2014.

[8] Gaoni Y, Mechoulam R. Isolation, structure, and partial synthesis of an active constituent of Hashish. J Am Chem Soc 1964;86:1646-7. http://dx.doi.org/ 10.1021/ja01062046.

[9] Pisanti S, Malfitano AM, Ciaglia E, Lamberti A, Ranieri R, Cuomo G, et al. Cannabidiol: state of the art and new challenges for therapeutic applications. Pharmacol Ther 2017;175:133-50. http://dx.doi.org/10.1016/j.pharmthera.2017.02.041.

[10] Néfau T. Le point SINTES - numéro 4. WwwOfdtFr; 2019 [accessed July 30 2019]https://www.ofdt.fr/BDD/sintes/LePointSINTES04.pdf.

[11] Clarke RC, Watson DP. Cannabis and natural cannabis medicines. In: ElSohly MA, editor. Marijuana and the Cannabinoids. Totowa, NJ: Humana Press; 2007. p. 1-15. http://dx.doi.org/10.1007/978-1-59259-947-9_1.

[12] Blackard C, Tennes K. Human placental transfer of cannabinoids. N Engl J Med 1984;311:797. http://dx.doi.org/10.1056/NEJM198409203111213.

[13] Bailey JR, Cunny HC, Paule MG, Slikker W. Fetal disposition of delta 9tetrahydrocannabinol (THC) during late pregnancy in the rhesus monkey. Toxicol Appl Pharmacol 1987;90:315-21. http://dx.doi.org/10.1016/0041008x(87)90338-3.

[14] Elphick MR. The evolution and comparative neurobiology of endocannabinoid signalling. Philos Trans R Soc Lond B Biol Sci 2012;367:3201-15. http:/ dx.doi.org/10.1098/rstb.2011.0394.

[15] Lutz B, Marsicano G, Maldonado R, Hillard CJ. The endocannabinoid system in guarding against fear, anxiety and stress. Nat Rev Neurosci 2015;16:705-18. http://dx.doi.org/10.1038/nrn4036.

[16] Ligresti A, De Petrocellis L, Di Marzo V. From phytocannabinoids to cannabinoid receptors and endocannabinoids: pleiotropic physiological and pathological roles through complex pharmacology. Physiol Rev 2016;96:1593-659. http://dx.doi.org/10.1152/physrev.00002.2016.

[17] Mechoulam R, Fride E, Di Marzo V. Endocannabinoids. Eur J Pharmacol 1998;359:1-18.

[18] Ueda N, Tsuboi K, Uyama T. Metabolism of endocannabinoids and related Nacylethanolamines: canonical and alternative pathways. FEBS J 2013;280: 1874-94. http://dx.doi.org/10.1111/febs.12152.

[19] Chicca A, Marazzi J, Nicolussi S, Gertsch J. Evidence for bidirectional endocannabinoid transport across cell membranes. J Biol Chem 2012;287:3466082. http://dx.doi.org/10.1074/jbc.M112.373241.

[20] Ueda N, Tsuboi K, Uyama T, Ohnishi T. Biosynthesis and degradation of the endocannabinoid 2-arachidonoylglycerol. BioFactors 2011;37:1-7. http: dx.doi.org/10.1002/biof.131.

[21] Howlett AC. Cannabinoid receptor signaling. In: Pertwee RG, editor. Handbook of experimental pharmacology, 168. Berlin/Heidelberg: Springer-Verlag; 2005. p. 53-79. http://dx.doi.org/10.1007/3-540-26573-2_2.

[22] Pearson G, Robinson F, Gibson TB, Xu B-E, Karandikar M, Berman K, et al. Mitogen-Activated Protein (MAP) Kinase Pathways. Regulat Physiol Funct 2001;22:31

[23] Almada M, Fonseca BM, Amaral C, Diniz-da-Costa M, Correia-da-Silva C Teixeira N. Anandamide oxidative metabolism-induced endoplasmic reticulum stress and apoptosis. Apoptosis 2017;22:816-26. http://dx.doi.org 10.1007/s10495-017-1356-4.

[24] Pertwee RG, Howlett AC, Abood ME, Alexander SPH, Di Marzo V, Elphick MR et al. International union of basic and clinical pharmacology. LXXIX. Cannabinoid receptors and their ligands: beyond CB1 and CB2. Pharmacol Rev 2010;62:588-631. http://dx.doi.org/10.1124/pr.110.003004.

[25] Diez-Alarcia R, Ibarra-Lecue I, Lopez-Cardona AP, Meana J, Gutierrez-Adán A, Callado LF, et al. Biased agonism of three different cannabinoid receptor agonists in mouse brain cortex. Front Pharmacol 2016;7. http://dx.doi.org 10.3389/fphar.2016.00415.

[26] Cravatt BF, Giang DK, Mayfield SP, Boger DL, Lerner RA, Gilula NB. Molecular characterization of an enzyme that degrades neuromodulatory fatty-acid amides. Nature 1996;384:83-7. http://dx.doi.org/10.1038/384083a0.
[27] Brown QL, Sarvet AL, Shmulewitz D, Martins SS, Wall MM, Hasin DS. Trends in marijuana use among pregnant and nonpregnant reproductive-aged women, 2002-2014. JAMA 2017;317:207-9. http://dx.doi.org/10.1001/ jama.2016.17383.

[28] Oh S, Salas-Wright CP, Vaughn MG, DiNitto DM. Marijuana use during pregnancy: a comparison of trends and correlates among married and unmarried pregnant women. Drug Alcohol Dependence 2017;181:229-33. http:// dx.doi.org/10.1016/j.drugalcdep.2017.09.036.

[29] El Marroun H, Tiemeier $\mathrm{H}$, Jaddoe VWV, Hofman A, Mackenbach JP, Steegers EAP, et al. Demographic, emotional and social determinants of cannabis use in early pregnancy: the Generation $R$ study. Drug Alcohol Dependence 2008;98:218-26. http://dx.doi.org/10.1016/j.drugalcdep.2008.05.010.

[30] Ko JY, Farr SL, Tong VT, Creanga AA, Callaghan WM. Prevalence and patterns of marijuana use among pregnant and nonpregnant women of reproductive age. Am J Obstet Gynecol 2015;213:201.e1-201.e10. http://dx.doi.org/10.1016/ j.ajog.2015.03.021.

[31] Chiandetti A, Hernandez G, Mercadal-Hally M, Alvarez A, Andreu-Fernandez V, Navarro-Tapia E, et al. Prevalence of prenatal exposure to substances of abuse: questionnaire versus biomarkers. Reprod Health 2017;14:137. http:// dx.doi.org/10.1186/s12978-017-0385-3.

[32] Young-Wolff KC, Tucker L-Y, Alexeeff S, Armstrong MA, Conway A, Weisner C, et al. Trends in self-reported and biochemically tested marijuana use among pregnant females in California from 2009-2016. JAMA 2017;318:2490. http:// dx.doi.org/10.1001/jama.2017.17225.

[33] Mark K, Gryczynski J, Axenfeld E, Schwartz RP, Terplan M. Pregnant women's current and intended cannabis use in relation to their views toward legalization and knowledge of potential harm. J Addict Med 2017;11:211-6. http:// dx.doi.org/10.1097/ADM.0000000000000299.

[34] Beatty JR, Svikis DS, Ondersma SJ. Prevalence and perceived financial costs of marijuana versus tobacco use among urban low-income pregnant women. J Addict Res Ther 2012;3. http://dx.doi.org/10.4172/2155-6105.1000135.

[35] Jarlenski M, Koma JW, Zank J, Bodnar LM, Bogen DL, Chang JC. Trends in perception of risk of regular marijuana use among US pregnant and nonpregnant reproductive-aged women. Am J Obstet Gynecol 2017;217:705-7. http:// dx.doi.org/10.1016/j.ajog.2017.08.015.

[36] El-Talatini MR, Taylor AH, Elson JC, Brown L, Davidson AC, Konje JC. Localisation and function of the endocannabinoid system in the human ovary. PLoS ONE 2009;4:e4579. http://dx.doi.org/10.1371/journal.pone.0004579.

[37] Schmid PC, Paria BC, Krebsbach RJ, Schmid HH, Dey SK. Changes in anandamide levels in mouse uterus are associated with uterine receptivity for embryo implantation. Proc Natl Acad Sci USA 1997;94:4188-92. http://dx.doi.org/ 10.1073/pnas.94.8.4188.

[38] Park B, McPartland JM, Glass M. Cannabis, cannabinoids and reproduction. Prostaglandins Leukotrienes and Essential Fatty Acids 2004;70:189-97. http://dx.doi.org/10.1016/j.plefa.2003.04.007.

[39] Wenger T, Tóth BE, Martin BR. Effects of anandamide (endogen cannabinoid) on anterior pituitary hormone secretion in adult ovariectomized rats. Life Sci 1995;56:2057-63. http://dx.doi.org/10.1016/0024-3205(95)00189-D.

[40] Di Blasio AM, Vignali M, Gentilini D. The endocannabinoid pathway and the female reproductive organs. J Mol Endocrinol 2013;50:R1-9. http://dx.doi.org/ 10.1530/JME-12-0182.

[41] Almirez RG, Smith CG, Asch RH. The effects of marijuana extract and delta 9tetrahydrocannabinol on luteal function in the rhesus monkey. Fertil Steril 1983;39:212-7.

[42] Chakravarty I, Shah PG, Sheth AR, Ghosh JJ. Mode of action of delta-9-tetrahydrocannabinol on hypothalamo-pituitary function in adult female rats. Reproduction 1979;57:113-5. http://dx.doi.org/10.1530/jrf.0.0570113.

[43] Asch RH, Smith CG, Siler-Khodr TM, Pauerstein CJ. Effects of delta 9-tetrahydrocannabinol during the follicular phase of the rhesus monkey (Macaca mulatta). J Clin Endocrinol Metab 1981;52:50-5. http://dx.doi.org/10.1210/ jcem-52-1-50.

[44] Mueller BA, Daling JR, Weiss NS, Moore DE. Recreational drug use and the risk of primary infertility. Epidemiology 1990;1:195-200.

[45] Klonoff-Cohen HS, Natarajan L, Victoria Chen R. A prospective study of the effects of female and male marijuana use on in vitro fertilization (IVF) and gamete intrafallopian transfer (GIFT) outcomes. Am J Obstet Gynecol 2006;194:369-76. http://dx.doi.org/10.1016/j.ajog.2005.08.020.

[46] Mendelson JH, Mello NK, Ellingboe J, Skupny AS, Lex BW, Griffin M. Marihuana smoking suppresses luteinizing hormone in women. J Pharmacol Exp Ther 1986;237:862-6.

[47] Sharma R, Biedenharn KR, Fedor JM, Agarwal A. Lifestyle factors and reproductive health: taking control of your fertility. Reprod Biol Endocrinol 2013;11:66. http://dx.doi.org/10.1186/1477-7827-11-66.

[48] Walker OS, Holloway AC, Raha S. The role of the endocannabinoid system in female reproductive tissues. J Ovarian Res 2019;12:3. http://dx.doi.org/ 10.1186/s13048-018-0478-9.

[49] Wang H, Guo Y, Wang D, Kingsley PJ, Marnett LJ, Das SK, et al. Aberrant cannabinoid signaling impairs oviductal transport of embryos. Nat Med 2004;10:1074-80. http://dx.doi.org/10.1038/nm1104.

[50] Wang H, Xie H, Guo Y, Zhang H, Takahashi T, Kingsley PJ, et al. Fatty acid amide hydrolase deficiency limits early pregnancy events. J Clin Invest 2006;116: 2122-31. http://dx.doi.org/10.1172/JCI28621.

[51] Gebeh AK, Willets JM, Marczylo EL, Taylor AH, Konje JC. Ectopic pregnancy is associated with high anandamide levels and aberrant expression of FAAH and CB1 in fallopian tubes. J Clin Endocrinol Metabol 2012;97:2827-35. http:// dx.doi.org/10.1210/jc.2012-1780. 
[52] Matsumoto H. Molecular and cellular events during blastocyst implantation in the receptive uterus: clues from mouse models. J Reprod Dev 2017;63:445-54. http://dx.doi.org/10.1262/jrd.2017-047.

[53] Brents LK. Marijuana, the endocannabinoid system and the female reproductive system. Yale J Biol Med 2016;89:175-91.

[54] Maccarrone M, Valensise H, Bari M, Lazzarin N, Romanini C, Finazzi-Agrò A. Relation between decreased anandamide hydrolase concentrations in human lymphocytes and miscarriage. Lancet 2000;355:1326-9. http://dx.doi.org/ 10.1016/S0140-6736(00)02115-2.

[55] El-Talatini MR, Taylor AH, Konje JC. The relationship between plasma levels of the endocannabinoid, anandamide, sex steroids, and gonadotrophins during the menstrual cycle. Fertil Steril 2010;93:1989-96. http://dx.doi.org/10.1016/ j.fertnstert.2008.12.033.

[56] Costa MA, Fonseca BM, Teixeira NA, Correia-da-Silva G. The endocannabinoid anandamide induces apoptosis in cytotrophoblast cells: involvement of both mitochondrial and death receptor pathways. Placenta 2015;36:69-76. http:// dx.doi.org/10.1016/j.placenta.2014.10.011.

[57] Costa MA, Keating E, Fonseca BM, Teixeira NA, Correia-da-Silva G. 2-Arachidonoylglycerol impairs human cytotrophoblast cells syncytialization: influence of endocannabinoid signalling in placental development. Mol Cellular Endocrinol 2015;399:386-94. http://dx.doi.org/10.1016/ j.mce.2014.09.005.

[58] Costa MA, Fonseca BM, Marques F, Teixeira NA, Correia-da-Silva G. The psychoactive compound of Cannabis sativa, $\Delta 9$-tetrahydrocannabinol (THC) inhibits the human trophoblast cell turnover. Toxicology 2015;334:94-103. http://dx.doi.org/10.1016/i.tox.2015.06.005.

[59] Almada M, Costa L, Fonseca BM, Amaral C, Teixeira N, Correia-da-Silva G. The synthetic cannabinoid WIN-55,212 induced-apoptosis in cytotrophoblasts cells by a mechanism dependent on CB1 receptor. Toxicology 2017;385: 67-73. http://dx.doi.org/10.1016/j.tox.2017.04.013.

[60] Gunn JKL, Rosales CB, Center KE, Nuñez A, Gibson SJ, Christ C, et al. Prenatal exposure to cannabis and maternal and child health outcomes: a systematic review and meta-analysis. BMJ Open 2016;6:e009986. http://dx.doi.org/ 10.1136/bmjopen-2015-009986.

[61] Conner SN, Bedell V, Lipsey K, Macones GA, Cahill AG, Tuuli MG. Maternal marijuana use and adverse neonatal outcomes: a systematic review and meta-analysis. Obstet Gynecol 2016;128:713-23. http://dx.doi.org/10.1097/ AOG.0000000000001649.

[62] Coleman-Cowger VH, Oga EA, Peters EN, Mark K. Prevalence and associated birth outcomes of co-use of Cannabis and tobacco cigarettes during pregnancy. Neurotoxicol Teratol 2018;68:84-90. http://dx.doi.org/10.1016/j.ntt.2018.06.001.

[63] Chamley LW, Bhalla A, Stone PR, Liddell H, O'Carroll S, Kearn C, et al. Nuclear localisation of the endocannabinoid metabolizing enzyme fatty acid amide hydrolase (FAAH) in invasive trophoblasts and an association with recurrent miscarriage. Placenta 2008;29:970-5. http://dx.doi.org/10.1016/j.placenta.2008.08.003.
[64] Hofman A, Jaddoe VWV, Mackenbach JP, Moll HA, Snijders RFM, Steegers EAP, et al. Growth, development and health from early fetal life until young adulthood: the Generation R Study. Paediatr Perinat Epidemiol 2004;18: 61-72.

[65] Hayatbakhsh MR, Flenady VJ, Gibbons KS, Kingsbury AM, Hurrion E, Mamun AA, et al. Birth outcomes associated with cannabis use before and during pregnancy. Pediatr Res 2012;71:215-9. http://dx.doi.org/10.1038/pr.2011.25.

[66] Leemaqz SY, Dekker GA, McCowan LM, Kenny LC, Myers JE, Simpson NAB, et al. Maternal marijuana use has independent effects on risk for spontaneous preterm birth but not other common late pregnancy complications. Reprod Toxicol 2016;62:77-86. http://dx.doi.org/10.1016/j.reprotox.2016.04.021.

[67] Saurel-Cubizolles M-J, Prunet C, Blondel B. Cannabis use during pregnancy in France in 2010. BJOG 2014;121:971-7. http://dx.doi.org/10.1111/14710528.12626.

[68] Dekker GA, Lee SY, North RA, McCowan LM, Simpson NAB, Roberts CT. Risk factors for preterm birth in an international prospective cohort of nulliparous women. PLoS ONE 2012;7:e39154. http://dx.doi.org/10.1371/journal.pone.0039154.

[69] Hatch EE, Bracken MB. Effect of marijuana use in pregnancy on fetal growth. Am J Epidemiol 1986;124:986-93. http://dx.doi.org/10.1093/oxfordjournalS.aje.a114488.

[70] Kliegman RM, Madura D, Kiwi R, Eisenberg I, Yamashita T. Relation of maternal cocaine use to the risks of prematurity and low birth weight. J Pediatrics 1994;124:751-6. http://dx.doi.org/10.1016/S0022-3476(05)81370-8.

[71] Shiono PH, Klebanoff MA, Nugent RP, Cotch MF, Wilkins DG, Rollins DE, et al The impact of cocaine and marijuana use on low birth weight and preterm birth: a multicenter study. Am J Obstet Gynecol 1995;172:19-27. http:/ dx.doi.org/10.1016/0002-9378(95)90078-0.

[72] van Gelder MMHJ, Reefhuis J, Caton AR, Werler MM, Druschel CM, Roeleveld N. Characteristics of pregnant illicit drug users and associations between cannabis use and perinatal outcome in a population-based study. Drug Alcohol Dependence 2010;109:243-7. http://dx.doi.org/10.1016/j.drugalcdep.2010.01.007.

[73] Warshak CR, Regan J, Moore B, Magner K, Kritzer S, Van Hook J. Association between marijuana use and adverse obstetrical and neonatal outcomes. J Perinatol 2015;35:991-5. http://dx.doi.org/10.1038/jp.2015.120.

[74] Metz TD, Allshouse AA, Hogue CJ, Goldenberg RL, Dudley DJ, Varner MW, et al. Maternal marijuana use, adverse pregnancy outcomes, and neonatal morbidity. Am J Obstet Gynecol 2017;217:478.e1-8. http://dx.doi.org/10.1016/ j.ajog.2017.05.050.

[75] Sun X, Dey SK. Endocannabinoid signaling in female reproduction. ACS Chem Neurosci 2012;3:349-55. http://dx.doi.org/10.1021/cn300014e.

[76] Richardson KA, Hester AK, McLemore GL. Prenatal cannabis exposure - The "first hit" to the endocannabinoid system. Neurotoxicol Teratol 2016;58:5-14 http://dx.doi.org/10.1016/j.ntt.2016.08.003.

[77] Fleming TP, Velazquez MA, Eckert JJ. Embryos, DOHaD and David Barker. J Dev Origins Health Dis 2015;6:377-83. http://dx.doi.org/10.1017/ $\underline{\text { S2040174415001105 }}$ 